\title{
Problematic Use of the Internet and Self-Regulation: A Review of the Initial Studies
}

\author{
Joël Billieux ${ }^{*}, 1,2$ and Martial Van der Linden ${ }^{1,3}$ \\ ${ }^{I}$ Cognitive Psychopathology and Neuropsychology Unit, University of Geneva, Geneva, Switzerland \\ ${ }^{2}$ Psychological Sciences Research Institute, Catholic University of Louvain, Louvain-La-Neuve, Belgium \\ ${ }^{3}$ Cognitive Psychopathology Unit, University of Liège, Liège, Belgium
}

\begin{abstract}
Problematic Internet Use (PIU) is considered as an inability to control one's use of the Internet, which eventually involves negative consequences in daily life. Among the various psychological factors potentially involved in the development of PIU, the role of poor self-regulation capacities (e.g., high impulsivity and sensation seeking, low inhibitory control, poor decision-making abilities) has recently received increased attention. Although the number of studies currently available on this topic remains limited, our aim here is to review their findings. After briefly defining PIU and discussing the main instruments which have been developed to assess it, this article describes the studies that have investigated the relations between PIU and self-regulation. Their heterogeneous findings are discussed and avenues for future research are provided.
\end{abstract}

Keywords: Internet, addiction, self-regulation, self-control, impulsivity, sensation seeking, UPPS.

\section{INTRODUCTION}

The use of the Internet has increased considerably over the last years and it has become an essential channel in domains such as social communication, academic research, and entertainment. Despite its recognized positive consequences for everyone's life, a growing number of studies have revealed that the use of the Internet can, in certain cases, become problematic [1,2].

Problematic Internet Use (PIU) is considered as an inability to control use of the Internet, which eventually involves psychological, social, academic, and/or professional problems in a person's life [3, 4]. PIU has been related to a variety of different activities such as cybersex (i.e., compulsive consumption of adult pornographic websites [5]), online gambling [6], and online video game playing [7], thereby emphasizing that this problematic behavior can take very different forms across individuals and should not be viewed as homogeneous. Research into the risk factors for PIU has emphasized that its occurrence may be influenced by demographics (e.g., gender, socio-economic status), psychological factors (e.g., personality traits, self-esteem, cognitive processes, motives for using the Internet), and comorbidity of concurrent symptoms (e.g., anxiety, depression, social phobia, drug use) [1,2].

Among the various psychological factors potentially implicated in PIU, a growing number of studies have explored the role of individual differences in self-regulation (e.g., traits of impulsivity and sensation seeking, inhibitory control and other executive processes, reinforcement

\footnotetext{
*Address correspondence to this author at the Psychological Sciences Research Institute, Catholic University of Louvain. 10, Place du Cardinal Mercier, 1348 Louvain-La-Neuve, Belgium; Tel : +32 104792 16;
} Fax: +32 104737 74; E-mail: Joël.Billieux@uclouvain.be sensitivity, emotion-regulation abilities). This focus on selfregulation is mainly due to the fact that PIU has often been conceptualized as an addiction and individual differences in self-regulation have been shown to play a crucial role in the development and maintenance of addictive behaviors, whether or not they are related to substance use [8]. Although several studies have found clear relations between PIU and low self-regulation (e.g., high impulsivity and sensation seeking, poor inhibitory control), the results of these studies often contribute little to our understanding of PIU as many of them have been carried out without a specific theoretical rational and/or have not considered the multifaceted nature of self-regulation-related processes. Bearing these limitations in mind, our aim here is to present an exhaustive and critical examination of these studies.

To this end, this article is divided into three distinct parts. The first section discusses how PIU has been conceptualized and defined and also describes the major instruments developed to assess it. The second section consists of a detailed presentation of the various studies of the relations between PIU and self-regulation. Finally, the third part summarizes the findings about PIU and self-regulation and describes a multifaceted model of impulsivity which could be of interest for further research on this topic: the UPPS impulsivity model [9].

\section{DEFINITION AND ASSESSMENT OF PROBLE- MATIC INTERNET USE}

Since its appearance in the medical and psychological literature, PIU has been viewed as a mental disorder and termed "Internet addiction" or "pathological Internet use" [4, $10,11]$. Consequently, PIU has been conceptualized from a categorical perspective inspired by the diagnostic criteria for certain addictive behaviors in the Diagnostic and Statistical Manual for Mental Disorders (DSM), such as substance 
abuse or pathological gambling. Thus, PIU is frequently considered as a "behavioral addiction" or an "impulse control disorder," sharing most features (e.g., craving, tolerance, loss of control, relapse, and withdrawal) with other addictive disorders. In this vein, several authors have proposed specific diagnostic criteria to identify "pathological Internet users" or "Internet addicts" $[3,12]$. There are even a growing number of calls for its inclusion in the future DSM$\mathrm{V}$ [13].

Several instruments have been developed to assess PIU. The most frequently used ones are diagnostic screening questionnaires aiming to distinguish "Internet addicts" from "normal Internet users," such as Young's eight-item questionnaire [14] inspired by the DSM-IV criteria for pathological gambling and the modified version by Beard and Wolf [12] (see Table 1). However, this type of assessment method has been criticized. For example, Dowling and Quirk [15] showed that Young's criteria do not adequately discriminate Internet addicts from "at risk" subjects (i.e., persons who report problems with their use of the Internet without reaching the proposed criteria for pathological Internet use). Thus, one should be aware that such types of categorical diagnostic criteria hinder consideration of the possibility that there is a continuum ranging from non-problematic high engagement in the Internet to PIU $[16,17]$.

Table 1. Criteria for Internet Addiction According to Beard and Wolf (2001)

The Five Necessary Criteria:

1. Is preoccupied with the Internet (thinking about previous online activity or anticipating next online session).

2. Needs to use the Internet for increased amounts of time in order to achieve satisfaction.

3. Makes unsuccessful efforts to control, cut back, or stop Internet use.

4. Is restless, moody, depressed, or irritable when attempting to cut down or stop Internet use.

5. Stays online longer than originally intended.

One of the Following Criteria is Necessary:

1. Has jeopardized or risked the loss of a significant relationship, job, educational or career opportunity because of the Internet.

2. Has lied to family members or someone else to conceal the extent of involvement with the Internet.

3. Uses the Internet as a way of escaping from problems or of relieving a dysphoric mood (e.g., feeling of helplessness, guilt, anxiety, depression).

Several self-report questionnaires have also been developed to measure PIU. Among them, the most frequently used is the Internet Addiction Test (IAT) [3], which comprises 20 different items assessing the negative consequences of overuse of the Internet (compromised social and individual quality of life, compensatory use of the Internet, compromised scholarly/academic/working career, compromised time control, excitatory use of the Internet). Although the development of the IAT was inspired by the diagnostic criteria for Internet Addiction, this questionnaire allows the severity of PIU to be considered, as the various items are scored on a Likert scale (ranging from "never" to "always") rather than on the basis of a "yes" or "no" answer.
The original IAT has been shown to have adequate psychometric properties [18] and has since been translated and validated in several other languages [19-21]. Other questionnaires have also been developed to investigate PIU, such as the Online Cognition Scale [22], the Generalized Problematic Internet Use Scale [23], and the Compulsive Internet Use Scale [24]. Self-reported questionnaires have also been developed to measure problematic involvement in a specific activity on the Internet. An example of such a questionnaire is the Online Games Addiction Scale [25], an adaptation of the IAT developed to target problematic involvement in online games, rather than PIU in general. However, one should be aware that these various scales have only received limited psychometric validation (e.g., their factorial structure has been determined by using exploratory techniques but not confirmatory techniques) [26]. Consequently, further validation studies are necessary to improve the psychometric properties of most of these scales.

To sum up, although PIU has received a growing amount of interest in this last decade, the construct itself has not been uniformly conceptualized across studies (e.g., there is no agreement concerning the diagnostic criteria for a potential "Internet addiction" entity). However, the investigation of PIU has to be considered as a recent topic, and more cumulative research and theoretical elaboration are needed to achieve a more unified view of this construct.

\section{SELF-REGULATION AND PROBLEMATIC INTERNET USE}

In recent years, a growing number of studies have explored the role of individual differences in self-regulation in the development of PIU, mainly because PIU has been conceptualized as an addictive behavior and self-regulation has been shown to play a critical role in such disorders [8].

An important distinction has been made between automatic and controlled aspects of self-regulation [27]. Automatic aspects of self-regulation correspond to the reaction of motivational systems when faced with a relevant stimulus. Thus, if one considers the case of an individual experiencing problems with his use of the Internet, a relevant stimulus (e.g., seeing the computer when arriving at home, experiencing an irrelevant thought related to the use of the Internet) may be likely to automatically trigger the approach motivational system, which will modulate the various kinds of processing (perceptual, motor, etc.) involved in approach behaviors and reinforcement seeking. Controlled aspects of self-regulation, on the other hand, depend on the effectiveness of the executive processes (e.g., inhibition of prepotent responses, shifting, working memory updating) whereby an individual voluntarily influences his or her thoughts, behaviors, and emotions. Thus, an individual who plays online games every day when returning from work will have to use controlled self-regulation capacities when he or she wants to inhibit this behavior.

The next section of this review describes the results of studies concerning the relations between PIU and individual differences in automatic and controlled self-regulation, first considering data about controlled aspects of self-regulation and then those relating to automatic aspects of selfregulation. 


\subsection{Problematic Internet Use and Controlled Aspects of Self-Regulation}

Relations between PIU and controlled regulation have been explored on the one hand by using self-report questionnaires assessing impulsivity (high impulsivity is considered to result from weak self-regulation capacities), and on the other hand by using cognitive tasks that assess inhibition functions and decision-making abilities.

Several studies have investigated the relations between PIU and self-reported impulsivity, which has been related to a variety of behaviors that appear to be poorly conceived, prematurely expressed, unduly risky, inappropriate to the context, and that often result in negative consequences [28]. Impulsivity plays a critical role in many theoretical models of addictive behaviors and high levels of self-reported impulsivity have been found in substance-dependent persons [29] and pathological gamblers [30]. Concerning the relationship between PIU and self-reported impulsivity, which is generally assessed with widely used and validated self-report impulsivity scales such as the Barratt Impulsivity Scale [31] or the UPPS Impulsive Behavior Scale [9], it has been found that individuals considered as Internet addicts (on the basis of a structural clinical interview inspired by Young's diagnostic criteria) have higher levels of selfreported impulsivity than control participants [32], and that impulsivity is positively correlated with PIU (generally assessed by the IAT) in undergraduate students [33] and online gamers $[34,35]$. In addition, there is a positive association between impulsivity in children (assessed by the parents) and the severity of PIU measured by the IAT [36]. Finally, Everton, Mastrangelo, and Jolton [37] showed that persons with high levels of impulsivity are more prone to use their computer in an unproductive way when they are at work (e.g., sending personal emails, playing games).

Recently, some studies have focused on the relations between PIU and the ability to inhibit a prepotent response, that is, the capacity to deliberately control or suppress an automatic response [38]. Indeed, several studies have shown deficits in inhibitory control in other types of addictions such as substance use disorder [39] and pathological gambling [40]. In this vein, Cao et al., [32] and Sun et al., [41] have compared the performance of Internet addicts (based on the diagnostic criteria proposed by Young) and control participants on prepotent response inhibition. Surprisingly, Cao et al., [32] found Internet addicts to have a lower ability to inhibit a prepotent response than control participants, whereas Sun et al., [41] obtained the opposite result, namely better inhibition performance for the Internet addiction group than for the control participant group. These contradictory results could possibly be attributed to the fact that the two studies did not use the same laboratory task to assess prepotent response inhibition. Cao et al., [32] used a stopsignal task that seems more suited for assessing controlled or executive inhibition than the go / no-go task used by Sun et al., [41], which has been shown to rely more on automatic subcortical processes (see [42] for the differences between these two inhibitory tasks). Further research remains necessary to clarify the relationships between prepotent response inhibition and PIU (e.g., by using laboratory tasks involving Internet-related cues and not only neutral stimuli). Note that future research should also consider the role of prepotent inhibition in clinically relevant aspects of PIU such as relapse, as poor inhibition has been found to be related to higher relapse rates in detoxified alcoholics [43] and pathological gamblers [44].

Sun et al., [41] also explored the link between PIU and the ability to take into account future consequences of an action by using the Iowa Gambling Task (IGT) [45], a laboratory task that simulates decision-making in a context in which it is difficult to determine the consequences (positive or negative) of a choice. Decision-making impairments in the IGT have been found in both substance abusers [39] and pathological gamblers [40]. From this perspective, Sun et al., [41] think that a myopia regarding future negative consequences could explain why persons suffering from PIU pursue their use of the Internet (e.g., excessive online gaming, compulsive consumption of pornographic material, online gambling) even if such behaviors will probably have tangible negative consequences for their daily lives. The results of the Sun et al., study showed that persons considered to be Internet addicts on the basis of Young's criteria make worse decisions (reflected by more disadvantageous choices in the IGT) than control participants. Future research should investigate decisionmaking abilities in relation to specific clinically relevant aspects of PIU (e.g., the role of the ability to take into account long-term consequences of Internet use for various personal and/or social issues) rather than only comparing groups of Internet addicts to control participants.

\subsection{Problematic Internet Use and Automatic Aspects of Self-Regulation}

The relationship between PIU and automatic aspects of self-regulation has mainly been investigated by using sensation seeking questionnaires, such as the Zuckerman Sensation Seeking Scale [46]. Sensation seeking is defined as the tendency to seek various novel, complex and arousing sensory stimulations, and openness toward new experiences $[9,46]$. This dimension of temperament has been related to the activation of the approach motivational system [47] and is frequently explored in relation to both substance use [48] and pathological gambling [49]. Several studies have also investigated the relations between PIU and sensitivity to positive and negative reinforcement in general. These studies have used questionnaires assessing individual differences in reward drive and punishment sensitivity, such as the Behavioral Inhibition System/Behavioral Activation System Scale [50] or the Temperament and Character Inventory [51]. Studies of the relations between PIU and automatic aspects of self-regulation have obtained mixed results. Certain studies found that PIU is positively associated with sensation-seeking [52-54] and with reward sensitivity [55, 56], whereas other studies failed to find such associations $[33,35,57-59]$. Moreover, some studies even found PIU to be associated with low sensation seeking [52-54] and reward sensitivity [53]. Similarly, punishment sensitivity has been found to be related to PIU in certain studies $[53,56]$ and unrelated in others $[55,57]$.

In our view, the contradictory results of these studies may be explained by the fact that the questionnaires targeting sensation seeking probably do not assess how persons suffering from PIU seek stimulation or/and rewards. 
Interestingly, heterogeneous results have also been found concerning the relations between sensation seeking and pathological gambling [49], which suggests that classical sensation seeking questionnaires (e.g., the Sensation Seeking Scale), which often focus on risky activities such as extreme sports, are probably not suited to investigating sensationseeking or reinforcement sensitivity in persons suffering from excessive gambling or problematic use of the Internet.

In this context, the use of laboratory tasks specifically designed to target certain aspects of passive self-regulation could be of interest to better understand its role in PIU. Future research could, for example, investigate the relations between PIU and reinforcement sensitivity by using laboratory tasks measuring the tendency to make choices based on the avoidance of negative reinforcement or the search for positive reinforcement [60]. Moreover, research about PIU and passive regulation should also explore the role of automatic or implicit attitudes toward the Internet. This could be done by using implicit laboratory tasks such as the Implicit Association Task [61]. Several studies that investigated the relations between substance use and implicit attitudes found that a positive implicit attitude toward a substance was related to more frequent use of this substance [62]. Thus, it could be supposed that a positive implicit attitude toward the Internet (or a specific aspect of Internet use such as online gaming or gambling) is related to actual use. In this context, further research conducted with more appropriate questionnaires or with laboratory tasks remains necessary to clarify the associations between PIU and passive aspects of self-regulation.

\section{SUMMARY AND CONCLUSION}

In the present article, we have reviewed the available literature about the role of controlled and automatic aspects of self-regulation in PIU. Concerning controlled aspects of self-regulation, it appears that persons suffering from PIU are characterized by higher levels of impulsivity. In addition, it also appears that PIU is associated with a weakness of the psychological mechanisms involved in the controlled regulation of behavior (e.g., the ability to take into account the long-term consequences of an action, the capacity to inhibit prepotent responses), although this latter result must be confirmed by further research. The role of passive regulation in PIU is less clear. Indeed, several studies found a relation between the occurrence of PIU and certain aspects of the passive regulation of behavior (e.g., sensation seeking, reward drive, punishment sensitivity), whereas other studies did not. In our view, the heterogeneity of the data provided by the studies of the relations between PIU and selfregulation abilities can be attributed to both methodological and theoretical concerns.

From a methodological point of view, we think that the inconsistency of the relations between PIU and selfregulation may be a function of the instruments and/or tasks selected (e.g., self-report questionnaires used to assess passive aspects of self-regulation, prepotent inhibition tasks), and by the tendency to conduct studies by considering PIU from a categorical perspective. Indeed, it is not impossible that some of the studies incorporated control participants who had certain problems regulating their use of the Internet even though they did not meet the criteria to be considered as Internet addicts. Moreover, doing studies by comparing Internet addicts to control participants entails the consideration of PIU on a continuum ranging from high Internet engagement through problematic or pathological use of the Internet.

From a theoretical point of view, we argue that the majority of the studies investigating PIU and self-regulation were carried out without a specific theoretical rationale that takes into account the multidimensionality of self-regulation processes. Indeed, major advancements have been made in recent years in the conceptualization and definition of the various facets of impulsive behaviors (considered as manifestations of a lack of self-regulation). Accordingly, we think that further research into self-regulation and PIU may benefit from consideration of a recent multifaceted model of impulsivity: the UPPS impulsivity model developed by Whiteside and Lynam [9]. These authors clarified the construct of impulsivity by identifying four separate components associated with impulsive behaviors. These four facets of impulsivity, which are the bases for the creation of a scale called the UPPS Impulsive Behavior Scale, are as follows: (1) urgency, defined as the tendency to act rashly in intense emotional contexts; (2) premeditation, defined as the tendency to think and reflect on the consequences of an act before engaging in that act; (3) perseverance, defined as the ability to remain focused on a task that may be boring and/or difficult; and (4) sensation seeking, considered as a tendency to enjoy and pursue activities that are exciting, and openness to trying new experiences. Recently, Bechara and Van der Linden [63] have proposed that specific psychological mechanisms implicated in the automatic and controlled regulation of behaviors underlie the various components of impulsivity. Interestingly, two studies done with volunteer participants from the community have shown that certain facets of impulsivity are related to the psychological mechanisms that have been investigated in relation to PIU, namely the capacity to inhibit a prepotent response and the ability to take into account the future consequences of an action. More specifically, it has been shown that a poor ability to take into account the consequence of an action is associated with a low level of premeditation [64], whereas a difficulty inhibiting prepotent responses is associated with a high level of urgency [65]. Interestingly, high urgency and low premeditation have been highlighted as predictors of excessive involvement in massively multiplayer online role playing games [35], a behavior frequently linked to PIU [66]. Moreover, high urgency and low premeditation have also been associated with the occurrence of other behavioral addictions such as pathological gambling [67], compulsive buying [68], or problematic use of mobile phones [69]. Thus, we think that the UPPS model of impulsivity and the various psychological mechanisms underlying its components could be a relevant theoretical rationale for further investigating the role of self-regulation in PIU.

To conclude, we would like to emphasize that PIU currently remains a controversial construct. Indeed, although the majority of studies have considered PIU from a categorical perspective, no agreement exists concerning the criteria for a potential diagnosis of "Internet addiction," and there are no studies of PIU from a dimensional perspective. Indeed, most of the research currently available on PIU consists of cross-sectional/transversal designs in which the 
Internet addict is incorporated in a limited and specific category rather than viewed as an individual at a particular stage of the Internet user trajectory. For example, firm conclusions about the chronicity and the persistence of PIU require further longitudinal investigations of prospective data. In addition, further research should also take into account, in addition to the socio-demographic variables generally considered (e.g., age, gender, socio-economic status), the possible interactions with other psychological processes which are likely to influence self-regulation. For example, it has been shown that the various motivations related to the use of the Internet (e.g., socializing, immersion in a virtual world, relieving negative affects) may also influence PIU $[17,70]$. Thus, a better comprehension of the role of self-regulation in the development, maintenance, and relapse in PIU require both longitudinal research and the consideration of other psychological processes that may potentially be involved (e.g., motives for using the Internet).

\section{ACKNOWLEDGEMENT}

Declared none.

\section{CONFLICT OF INTEREST}

Declared none.

\section{REFERENCES}

[1] Widyanto L, Griffiths M. "Internet addiction": A critical review. Int J Ment Health Addict 2006; 4: 31-51.

[2] Chou C, Condron L, Belland JC. A review of the research on the Internet addiction. Educ Psychol Rev 2005; 17: 363-88.

[3] Young KS. Internet addictions: symptoms, evaluation and treatment. In: Van de Creek L, Jackson T, Eds. Innovations in clinical practice: a source book. Sarasota, FL: Professional Resource Press 1999; pp. 19-31.

[4] Shaffer HJ, Hall MN, Vander Bilt J. "Computer addiction": A critical consideration. Am J Orthopsychiatry 2000; 70: 162-8.

[5] Meerkerk GJ, Van den Eijnden, RJJM, Garretsen HFL. Predicting compulsive Internet use: it's all about sex. Cyberpsychol Behav 2006; 9: 95-103.

[6] Griffiths M. Internet gambling: issues, concerns, and recommendations. Cyberpsychol Behav 2003; 6: 557-68.

[7] Ng BD, Wiemer-Hastings P. Addiction to the Internet and online gaming. Cyberpsychol Behav 2005; 8: 110-3.

[8] Dawe S, Loxton NJ. The role of impulsivity in the development of substance use and eating disorders. Neurosci Biobehav Rev 2004; 28: $343-51$.

[9] Whiteside SP, Lynam DR. The five factor model and impulsivity: using a structural model of personality to understand impulsivity. Pers Indiv Diff 2001; 30: 669-89.

[10] Griffiths M. Internet addiction: fact or fiction? Psychologist 1999; 12: 246-50.

[11] Young KS. Caught in the net. New York: Wiley 1998.

[12] Beard KW, Wolf EM. Modification in the proposed diagnostic criteria for Internet addiction. Cyberpsychol Behav 2001; 4: 37783.

[13] Block JJ. Issues for DSM-V: Internet addiction. Am J Psychiatry 2008; 165: 306-7.

[14] Young KS. Internet addiction: the emergence of a new clinical disorder. Cyberpsychol Behav 1996; 3: 237-44.

[15] Dowling NA, Quirk KL. Screening for Internet dependence: Do the proposed diagnostic criteria differentiate normal from dependent Internet use? Cyberpsychol Behav 2009; 12: 21-7.

[16] Charlton JP, Danforth IDW. Distinguishing addiction and high engagement in the context of online game playing. Comput Hum Behav 2007; 23: 1531-48.

[17] LaRose R, Lin CA, Eastin MS. Unregulated Internet usage: addiction, habit, or deficient self-regulation? Media Psychol 2003; 5: 225-53.

[18] Widyanto L, McMurran M. The psychometric properties of the Internet Addiction Test. Cyberpsychol Behav 2004; 7: 443-50.
[19] Ferraro G, Caci B, D’Amico A, Di Blasi M. Internet addiction disorder: an Italian study. Cyberpsychol Behav 2007; 10: 170-5.

[20] Khazaal Y, Billieux J, Thorens G, et al. French validation of the Internet Addiction Test. Cyberpsychol Behav 2008; 11: 703-6.

[21] Kesici S, Sahin I. Turkish adaptation of the Internet Addiction Scale. Cyberpsychol Behav 2010; 13: 185-9.

[22] Davis RA. Validation of a new scale for measuring problematic Internet use: implication for pre-employment screening. Cyberpsychol Behav 2002; 5: 331-45.

[23] Caplan SE. Problematic Internet use and psycho-social well being: development of a theory based cognitive-behavioral measurement instrument. Comput Hum Behav 2002; 18: 553-75.

[24] Meerkerk GJ, Van den Eijnden RJJM, Vermulst AA, Garretsen HFL. The Compulsive Internet Use Scale (CIUS): some psychometric properties. Cyberpsychol Behav 2009; 12: 1-5.

[25] Whang LS, Chang GY. Psychological analysis of online game users. Proc Hum Comput Interact 2002; 2: 81-90.

[26] Jia R, Jia HH. Factorial validity of problematic Internet use scales. Comput Hum Behav 2009; 25: 1335-42.

[27] Derryberry D, Rothbart MK. Reactive and effortful processes in the organization of temperament. Dev Psychopathol 1997; 9: 633-52.

[28] Daruna JH, Barnes PA. A neurodevelopmental view of impulsivity. In: McCown WG, Johnson JL, Shure MB, Eds. The impulsive client: theory, research, and treatment. Washington DC: American Psychological Association 1993; pp. 23-37.

[29] Verdejo-García A, Bechara A, Recknor EC, Pérez-García M. Negative emotion-driven impulsivity predicts substance dependence problems. Drug Alcohol Dep 2007; 91: 213-9.

[30] Loxton NJ, Nguyen D, Casey L, Dawe S. Reward drive, rash impulsivity and punishment sensitivity in problem gamblers. Pers Indiv Diff 2008; 45: 167-73.

[31] Patton JH, Stanford MS, Barratt ES. Factor structure of the Barratt impulsiveness scale. J Clin Psychol 1995; 51: 768-74.

[32] Cao F, Su L, Liu T, Gao X. The relationship between impulsivity and Internet addiction in a sample of Chinese adolescents. Eur Psychiatry 2007; 22: 466-71.

[33] Mottram AJ, Fleming MJ. Extraversion, impulsivity, and online group membership as predictors of problematic Internet use. Cyberpsychol Behav 2009; 12: 319-21.

[34] Kim EJ, Namkoong K, Ku T, Kim SJ. The relationship between online game addiction and aggression, self-control and narcissistic personality traits. Eur Psychiatry 2008; 23: 212-8.

[35] Billieux J, Chanal J, Khazaal Y, et al. Psychological predictors of problematic involvement in Massively Multiplayer Online Role Playing Games (MMORPG): illustration in a sample of male cybercafés players. Psychopathology 2011; 44: 165-71.

[36] Yoo HJ, Cho SC, Ha JH, et al. Attention deficit hyperactivity symptoms and Internet addiction. Psychiat Clin Neurosci 2004; 58: 487-94.

[37] Everton W, Mastrangelo PM, Jolton JA. Personality correlates of employees' personal use of work computers. Cyberpsychol Behav 2005; 8: 143-53.

[38] Friedman NP, Miyake A. The relations among inhibition and interference control functions: a latent-variable analysis. J Exp Psychol Gen 2004; 133: 101-35.

[39] Noël X, Bechara A, Dan B, et al. Response inhibition deficit is involved in poor decision making under risk in nonamnesic individuals with alcoholism. Neuropsychology 2007; 21: 778-86.

[40] Goudriaan AE, Oosterlaan J, de Beurs E, van den Brink W. Decision making in pathological gambling: a comparison between pathological gamblers, alcohol dependents, persons with Tourette syndrome, and normal controls. Cognit Brain Res 2005; 23: 13751.

[41] Sun BDL, Chen ZJ, Ma N, et al. Decision-making and prepotent response inhibition functions in excessive Internet users. CNS Spectr 2009; 14: 75-81.

[42] Verbruggen F, Logan GD. Automatic and controlled response inhibition: associative learning in the go/no-go and stop-signal paradigms. J Exp Psychol Gen 2008; 137: 649-72.

[43] Noël X, Sferrazza R, Van der Linden M, et al. Contribution of frontal cerebral blood flow measured by ${ }^{99 \mathrm{~m}} \mathrm{Tc}$-Bicisate SPECT and executive function deficits to predicting treatment outcome in alcohol-dependent patients. Alcohol Alcohol 2002; 37: 347-54.

[44] Goudriaan AE, Oosterlaan J, de Beurs E, van den Brink W. The role of self-reported impulsivity and reward sensitivity $v s$ neurocognitive measures of disinhibition and decision-making in 
the prediction of relapse in pathological gamblers. Psychol Med 2008; 38: 41-50.

[45] Bechara A, Damasio AR, Damasio H, Anderson SW. Insensitivity to future consequences following damage to human prefrontal cortex. Cognition 1994; 50: 7-15.

[46] Zuckerman M. Behavioral expressions and biosocial bases of sensation seeking. New York: Cambridge University Press 1994

[47] Cloninger CR, Adolfsson R, Svrakic NM. Mapping genes for human personality. Nature Gen 1996; 12: 3-4.

[48] Roberti JW. A review of behavioral and biological correlates of sensation seeking. J Res Pers 2004; 38: 256-79.

[49] Hammelstein P. Faites vos jeux! Another look at sensation seeking and pathological gambling. Pers Indiv Diff 2004; 37: 917-31.

[50] Carver CS, White TL. Behavioural inhibition, behavioural activation, and affective responses to impending reward and punishment: the BIS/BAS scales. J Pers Soc Psychol 1994; 67: 319-33.

[51] Cloninger CR, Svrakic DM, Przybeck TR. A psychobiological model of temperament and character. Arch Gen Psychiatry 1993; 50: 975-90.

[52] Kim HK, Davis KE. Toward a comprehensive theory of problematic Internet use: evaluating the role of self-esteem, anxiety, flow, and the self-rated importance of Internet activities. Comput Hum Behav 2009; 25: 490-500.

[53] Ko $\mathrm{CH}$, Yen JY, Chen $\mathrm{CC}$, Chen $\mathrm{SH}$, Wu $\mathrm{K}$, Yen $\mathrm{CF}$. Tridimensional personality of adolescents with Internet addiction and substance use experience. Can J Psychiatry 2006; 51: 887-94.

[54] Lin SSJ, Tsai CC. Sensation seeking and Internet dependence of Taiwanese high school adolescents. Comput Hum Behav 2002; 18 : 411-26.

[55] Ko CH, Yen JY, Yen CF, Chen CH, Weng CC, Chen CC. The association between Internet addiction and problematic alcohol use in adolescents: The problem behavior model. Cyberpsychol Behav 2008; 11: 571-6.

[56] Yen JY, Ko CH, Yen CH, Cheng CS, Chen CC. The association between harmful alcohol use and Internet addiction among college students: comparison of personality. Psychiatry Clin Neurosci 2009; 63: 218-24.

[57] Cho SC, Kim JW, Kim BN, Lee JH, Kim EH. Biogenetic temperament and character profiles and attention deficit hyperactivity disorder symptoms in Korean adolescents with problematic Internet use. Cyberpsychol Behav 2008; 11: 735-7.

[58] Armstrong L, Phillips JG, Saling LL. Potential determinants of heavier Internet usage. Int J Hum Comput Stud 2000; 53: 537-50.

[59] Ha JH, Kim SY, Bae SC, et al. Depression and Internet addiction in adolescents. Psychopathology 2007; 40: 424-30.

[60] Franck MJ, Seeberger LC, O’Reilly RC. By carrot or by stick: cognitive reinforcement learning in Parkinsonism. Science 2004; 306: 1940-3.

[61] Greenwald AG, McGhee DE, Schwartz JLK. Measuring individual differences in implicit cognition: the implicit association test. J Pers Soc Psychol 1998; 74: 1464-80.

[62] Waters AJ, Carter BL, Robinson JD, Wetter DW, Lam CY, Cinciripini PM. Implicit attitudes to smoking is associated with craving and dependence. Drug Alcohol Dep 2007; 91: 178-86.

[63] Bechara A, Van der Linden M. Decision-making and impulse control after frontal lobe injuries. Curr Opin Neurol 2005; 18: 7349.

[64] Zermatten A, Van der Linden M, d'Acremont M, Jermann F, Bechara A. Impulsivity and decision making. J Nerv Ment Dis 2005; 93: 647-50.

[65] Gay P, Rochat L, Billieux J, d'Acremont M, Van der Linden M. Heterogeneous inhibition processes involved in different facets of self-reported impulsivity: evidence from a community sample. Acta Psychol 2008; 129: 332-9.

[66] Smyth J. Beyond self-selection in video game play: an experimental examination of the consequences of massively multiplayer online role-playing game play. Cyberpsychol Behav 2007; 10: 717-21.

[67] Smith GT, Fischer S, Cyders MA, Annus AM, Spillane NS, McCarthy DM. On the validity and utility of discriminating among impulsivity-like traits. Assessment 2007; 14: 155-70.

[68] Billieux J, Rochat L, Rebetez MML, Van der Linden M. Are all facets of impulsivity related to self-reported compulsive buying behavior? Pers Indiv Diff 2008; 44: 1432-42.

[69] Billieux J, Van der Linden M, Rochat L. The role of impulsivity in actual and problematic use of the mobile phone. Appl Cognit Psychol 2008; 2: 1195-210.

[70] Yee N. Motivations for play in online games. Cyberpsychol Behav 2006; 9: 772-5. 